\title{
Dark-field hyperlens for high-contrast sub-wavelength imaging
}

\author{
Repän, Taavi; Zhukovsky, Sergei; Lavrinenko, Andrei; Willatzen, Morten
}

\section{Published in:}

Proceedings of SPIE

Link to article, DOI:

$10.1117 / 12.2224720$

Publication date:

2016

Document Version

Publisher's PDF, also known as Version of record

Link back to DTU Orbit

Citation $(A P A)$ :

Repän, T., Zhukovsky, S., Lavrinenko, A., \& Willatzen, M. (2016). Dark-field hyperlens for high-contrast subwavelength imaging. In Proceedings of SPIE (Vol. 9883). [98830T] SPIE - International Society for Optical Engineering. Proceedings of SPIE - The International Society for Optical Engineering https://doi.org/10.1117/12.2224720

\section{General rights}

Copyright and moral rights for the publications made accessible in the public portal are retained by the authors and/or other copyright owners and it is a condition of accessing publications that users recognise and abide by the legal requirements associated with these rights.

- Users may download and print one copy of any publication from the public portal for the purpose of private study or research.

- You may not further distribute the material or use it for any profit-making activity or commercial gain

- You may freely distribute the URL identifying the publication in the public portal 


\title{
Dark-field hyperlens for high-contrast sub-wavelength imaging
}

\author{
Taavi Repän ${ }^{\mathrm{a}}$, Sergei Zhukovsky ${ }^{\mathrm{a}}$, Andrei Lavrinenko $^{\mathrm{a}}$, and Morten Willatzen ${ }^{\mathrm{a}}$

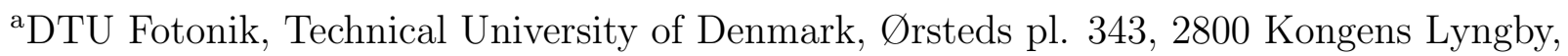 \\ Denmark
}

\begin{abstract}
By now superresolution imaging using hyperbolic metamaterial (HMM) structures - hyperlenses - has been demonstrated both theoretically and experimentally. The hyperlens operation relies on the fact that HMM allows propagation of waves with very large transverse wavevectors, which would be evanescent in common isotropic media (thus giving rise to the diffraction limit). However, nearly all hyperlenses proposed so far have been suitable only for very strong scatterers - such as holes in a metal film. When weaker scatterers, dielectric objects for example, are imaged then incident light forms a very strong background, and weak scatterers are not visible due to a poor contrast.

We propose a so-called dark-field hyperlens, which would be suitable for imaging of weakly scattering objects. By designing parameters of the HMM, we managed to obtain its response in such way that the hyperlens structure exhibits a cut-off for waves with small transverse wavevectors (low-k waves). This allows the structure to filter out the background illumination, which is contained in low-k waves. We numerically demonstrate that our device achieves superresolution imaging while providing the strong contrast for weak dielectric scatterers. These findings hold a great promise for dark-field superresolution, which could be important in real-time dynamic nanoscopy of label-free biological objects for example.
\end{abstract}

Keywords: hyperbolic metamaterials, hyperlens, superresolution, dark-field hyperlens

\section{INTRODUCTION}

Developments in the field of nanotechnology during the past 20 years have made it possible to experimentally study increasingly complex nanostructures. This has been a driving force behind the rise of a new field in nanooptics: metamaterials. By analogy to conventional materials - which are assembled of molecules and atoms - we can then build metamaterials out of suitably designed small unit cells (we could call them "meta-atoms"). ${ }^{1}$ As long as the size of these unit cells remains well below the wavelength, meaning that electric fields vary very little over a single unit cell, we can average electric fields over the unit cells and then in effective medium approximation the whole metamaterial can be represented as continuously homogeneous material described by effective material parameters.

It turns out that even relatively simple structures can give rise to very interesting phenomena: for example we can apply effective medium approach to a dielectric-metal multilayer, given that layer thicknesses remain subwavelength. Depending on optical parameters of the constituent materials the structure could end up having an "extreme" anisotropy - the components of permittivity tensor $\hat{\varepsilon}=\operatorname{diag}\left(\varepsilon_{\|}, \varepsilon_{\|}, \varepsilon_{\perp}\right)$ would have differing signs. Metamaterials with such permittivity tensor are called hyperbolic metamaterials (HMM). ${ }^{2}$

Although several methods of electron microscopy already give us a very good look into nanometer scale features, there is still a strong interest in optical imaging of small structures. However, resolution of conventional microscopes is limited by diffraction to a scale of a few hundred nanometers. There have been several ideas proposed to circumvent that limitation. A few of such - STED and SNOM for example - are already in

Further author information: (Send correspondence to T.R.)

T.R.: E-mail: tarap@fotonik.dtu.dk

A.V.: E-mail: alav@fotonik.dtu.dk

M.W.: E-mail: morwi@fotonik.dtu.dk

Metamaterials X, edited by Allan D. Boardman, Nigel P. Johnson, Kevin F. MacDonald, Ekmel Özbay, Proc. of SPIE Vol. 9883, 98830T · C 2016 SPIE · CCC code: 0277-786X/16/\$18 · doi: 10.1117/12.2224720 
mainstream use. The surge of metamaterials has of course given rise to new ideas for sub-wavelength imaging as well. One very well known example is Pendry's perfect lens, ${ }^{3}$ which utilizes double-negative metamaterials. However, the use of these metamaterials has many associated drawbacks and difficulties which has prevented real-world application of such designs. ${ }^{4}$ Nevertheless, it has inspired researchers to try to use metamaterials for sub-wavelength imaging. The hyperlens concept was first proposed ${ }^{4}$ in 2006 , where the authors suggested using hyperbolic metamaterials to image objects below diffraction limit. Since then hyperlensing has been also experimentally demonstrated ${ }^{5,6}$.

\section{THEORY}
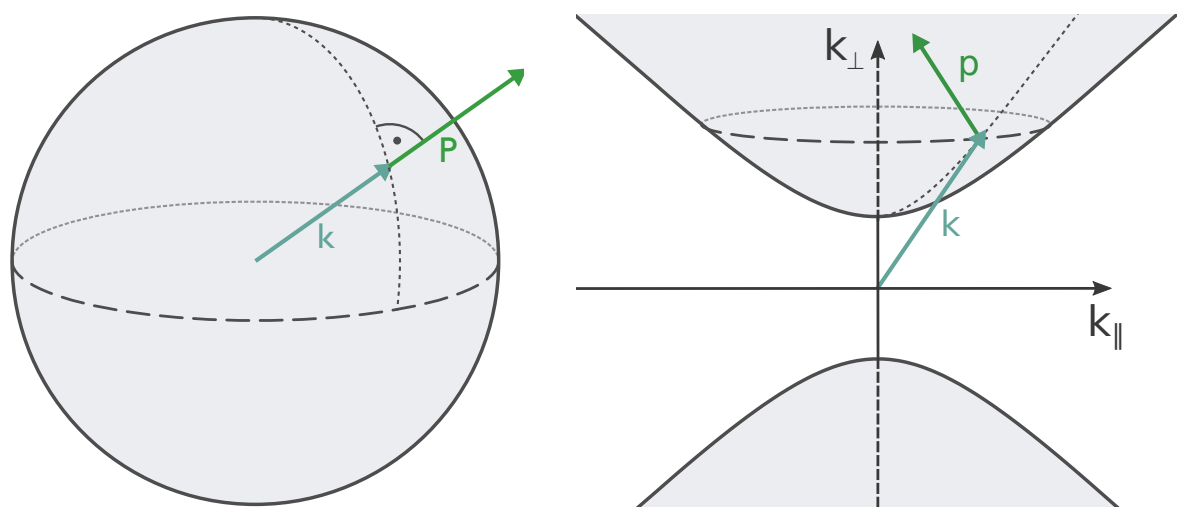

Figure 1. Isofrequency contours for isotropic media (sphere) and type-I HMM (hyperboloid). Also illustrated on the figure is a wavevector $(\mathrm{k})$ with a corresponding Poynting vector $(\mathrm{P})$. For an isotropic medium the both vectors are parallel, while in case of a hyperbolic media they can be perpendicular (as illustrated).

In a homogeneous uniaxial anisotropic medium the dispersion relation for plane waves of TM polarization is given by

$$
\frac{k_{\|}^{2}}{\varepsilon_{\perp}}+\frac{k_{\perp}^{2}}{\varepsilon_{\|}}=k_{0}^{2}
$$

where $k_{0}^{2}=\omega^{2} / c^{2}, \varepsilon_{\|}$and $\varepsilon_{\perp}$ are components of the permittivity tensor $\hat{\varepsilon}=\operatorname{diag}\left(\varepsilon_{\|}, \varepsilon_{\|}, \varepsilon_{\perp}\right)$ and $k_{\|}$and $k_{\perp}$ are respective components of the wavevector. In case of an isotropic medium the isofrequency surface reduces to a sphere, meaning that the magnitude of the wavevector is fixed and does not depend on propagation direction (see Fig. 1). The same figure also displays a well-known fact that for an isotropic medium the wavevector (determining the orientation of wavefronts) and Poynting vector (determining wave propagation direction) are parallel. However, for an anisotropic medium this does not hold anymore. In case of a conventional anisotropic medium the isofrequency surface forms an ellipsoid and in general the wavevector and the Poynting vector are not parallel.

In case of metamaterials a more drastic anisotropy can be achieved, where $\varepsilon_{\|}$and $\varepsilon_{\perp}$ have differing signs, in which case the isofrequency surface forms a hyperboloid. This gives such metamaterials the name hyperbolic metamaterials. Compared to an ordinary isotropic case (see Fig. 1) the hyperbolic metamaterial exhibits a completely new feature: the isofrequency surface is unbounded. This also means that there is no upper limit for wavevectors that are allowed to propagate. This in turn implies, that (at least in theory) there is no lower limit for the effective wavelength. In practice, of course, this holds only as far as the effective medium approximation goes - as the effective wavelength approaches the feature size of the structure then the approximation does not hold any more and at higher wavevectors the isofrequency surface deviates significantly from hyperboloid (actually forming a closed surface, as one would expect). ${ }^{7}$

In our notation we take $k_{\|}$to be the wavevector component parallel to the metamaterial interface and $k_{\perp}$ is the wavevector component normal to the metamaterial surface. We shall classify waves as low-k and high- $\mathrm{k}$ waves. By low-k waves we mean waves that can propagate in free space (being above diffraction limit), in other 
words $k_{\|}<k_{0}$. Conversely, by high-k waves we mean waves with $k_{\|}>k_{0}$, so that they would be evanescent in free space.

The sub-wavelength details of the image are contained in the high-k waves, which are evanescent in free space. Given that hyperbolic metamaterials are able to carry these high-k waves we can employ the hyperbolic metamaterials for sub-wavelength imaging. In order to couple the fields carrying sub-wavelength details to the far-field, the image must be magnified by a hyperlens above the diffraction limit. To achieve that a cylindrical geometry is used. Suppose we have some sub-wavelength sources - dipoles for example - on the inner interface of the hyperlens. The dipoles radiate waves with large span of k-vectors. In free space the high-k waves are attenuated (due to the diffraction limit). However the HMM allows propagation of such waves so they will be coupled into the hyperlens and thus will propagate through the device. As the image propagates through the hyperlens, it is magnified due to cylindrical geometry. ${ }^{4}$ At the outer interface a portion of those high-k waves will be magnified above the diffraction limit and therefore can be imaged using a conventional optical microscope.

One particularly noteworthy range of parameters for the hyperbolic metamaterial is when $\left|\varepsilon_{\perp}\right| \gg\left|\varepsilon_{\|}\right|$. This in turn means that (at least for small $k_{\|}$) the isofrequency surface will be flat and thus all waves will propagate in the same direction. This regime is known as the "canalization regime", ${ }^{8,9}$ reflecting the fact that fields from one interface of the metamaterial slab are "canalized" to the other interface. Since operating in this regime allows to achieve minimal image distortion (see Fig. 2), most hyperlenses demonstrated thus far have been designed to work in this regime.

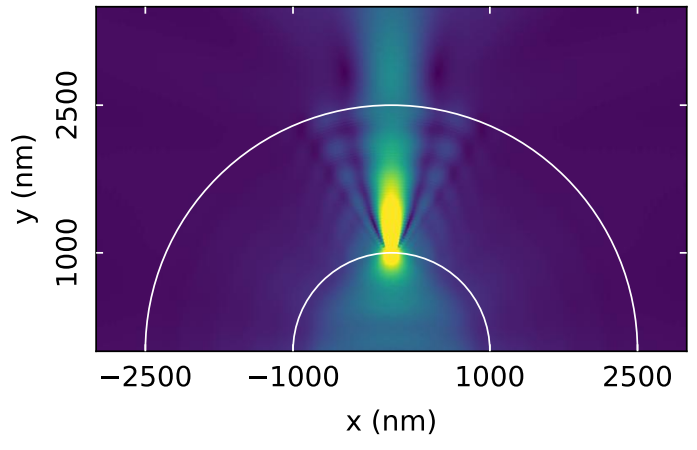

(a)

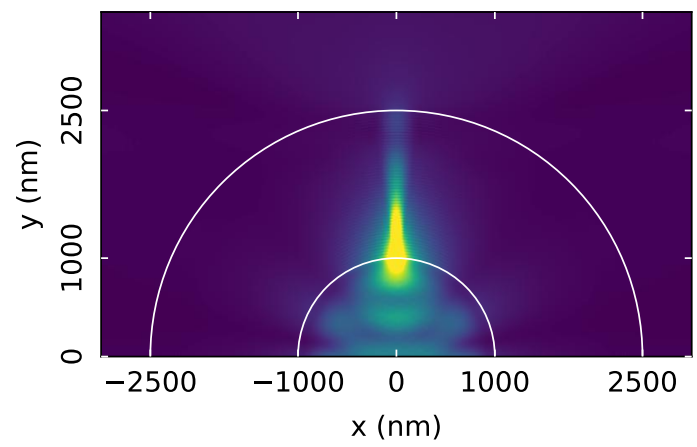

(b)

Figure 2. Bright field hyperlenses. a) A device operating away from the canalization regime - the beam from point source diverges while propagating through the hyperlens. b) A hyperlens operating in the canalization regime, showing narrow beam forming a tight spot on the output.

Experimental reports of hyperlenses so far have assumed very bright scatterers such as slits in a metal screen for example ${ }^{5}$. Since the illuminating fields pass through the hyperlens in the same way as scattered light does, use of bright objects is necessary - when imaging weak scatterers, strong background fields will strongly dominate the much weaker scattered image making it difficult to distinguish the imaged objects from the strong background.

In contrast to the usual hyperlens design, which could be labelled as the bright-field hyperlens, Benitsy, et al. ${ }^{10}$ coined the term dark-field hyperlens. They proposed a device, which uses a special geometry for illuminating objects so that coupling of the illuminating fields into the hyperlens (and therefore also to the output image) could be avoided. This allows to use the hyperlens for imaging weakly scattering objects, such as seen in biological imaging, for example.

Depending on signs of the permittivity tensor the HMM could be divided into two categories: type I and type II HMM. ${ }^{11}$ In context of bright-field hyperlenses the focus is on type I HMMs (shown in Fig. 1). This kind of HMM is obtained when $\varepsilon_{\perp}<0<\varepsilon_{\|}$. In the other case, $\varepsilon_{\perp}>0>\varepsilon_{\|}$, we get a type-II HMM. The resulting isofrequency surface is depicted in fig. 3. An important difference is that there exists a cut-off wavenumber $k_{c}$ : waves with $k_{\|}<k_{c}$ will not be allowed to propagate inside the type-II HMM. In our recent paper ${ }^{12}$ we proposed 
a dark-field hyperlens design where background illumination carried in the low-k waves is filtered by use of a type-II HMM in the hyperlens.

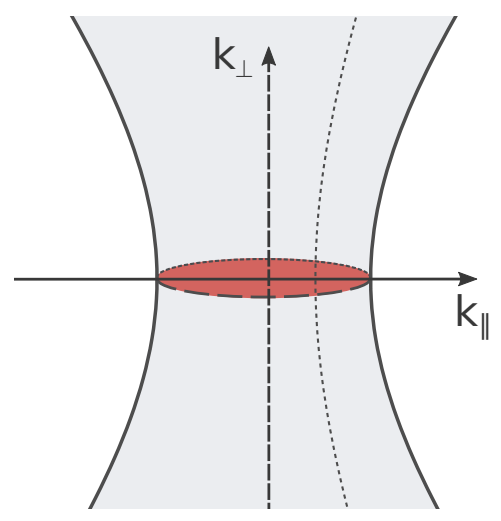

Figure 3. Isofrequency surface for a type-II HMM. The area with red shading indicates a band of waves, where $k_{\|}$is below the cut-off and therefore are not allowed to propagate in the medium.

Use of the type-II HMM in the hyperlens design however creates immediately new challenge - for type-II HMMs there isn't a comparable canalization regime. Indeed, when approaching the case where $\left|\varepsilon_{\perp}\right| \gg\left|\varepsilon_{\|}\right|$one observes that the attenuation of the low-k waves will tend towards zero: ${ }^{12}$ this means deterioration of dark-field operation of the hyperlens. As a consequence the dark-field hyperlens has to be designed to operate away from the canalization regime. However, the ratio of $\left|\varepsilon_{\perp}\right| /\left|\varepsilon_{\|}\right|$also determines the propagation angle of the waves in the hyperlens (wrt. the hyperlens surface normal) - as the ratio decreases the propagation angle of the waves increases. This means that there is a compromise between filtering of low-k waves and image distortion, since at the larger propagation angles the image will be more distorted.

Another characteristic of a type-II HMM based hyperlens is an upper limit for magnification. As the image travels through the cylindrical hyperlens it is slowly magnified, which corresponds of scaling $k_{\|}$to lower values. As the high-k waves propagate through the system they will (due to scaling of k-vectors) eventually move into the regime below low-k cut-off. This means that these waves will now be aggressively attenuated by the hyperbolic metamaterial. As a consequence at larger larger magnifications the hyperlens will only probe the higher wavevectors, as lower ones will enter into stop-band inside the hyperlens. However, waves with large wavenumbers are already weaker due to their stronger evanescent decay in the medium before the hyperlens. All in all this means that for hyperlens made of a single type-II HMM the magnification is effectively limited.

\section{RESULTS}

The basic design and material parameters are based on our earlier work. ${ }^{12}$ However, we have split our hyperlens into two parts: in the first part we work in the type-II HMM regime, which filters out the low-k background radiation. There we use a simple type-II HMM cylindrical slab composed of $2 \times 50$ layers with total thickness of $1500 \mathrm{~nm}$. The material parameters for metal and dielectric layers are $n_{m}=0.14+2.26 i$ (layer thickness 12 $\mathrm{nm}$ ) and $n_{d 1}=1.24$ (layer thickness $18 \mathrm{~nm}$ ). Second part consists of $2 \times 60$ layers, with different dielectric $\left(n_{d 2}=2.0\right)$. The layer thicknesses for dielectric layers has been changed to $48 \mathrm{~nm}$, while metal layers are still kept at $12 \mathrm{~nm}$. This second medium is not hyperbolic, but instead elliptic . This is actually desirable since the first part of the hyperlens has already filtered out the low-k waves, so there is no need for low-k filtering in the second part. This allows us to avoid the magnification limit of purely type-II HMM based hyperlens. Figure 4 shows the design of our hyperlens along with the dispersion relations for both parts of the structures. We note that this configuration also avoids out-coupling issues of our previous single metamaterial design.

To demonstrate sub-wavelength imaging capacity of the proposed hyperlens device we simulated two point sources placed near the inner interface of the hyperlens. We studied fields on the outer interface of the hyperlens as a function of the source separation. Resulting output fields from the hyperlens are plotted in Fig. 5. As can be seen from the figure, distance between the peaks is increased by a factor of 4.9 (as a consequence of 


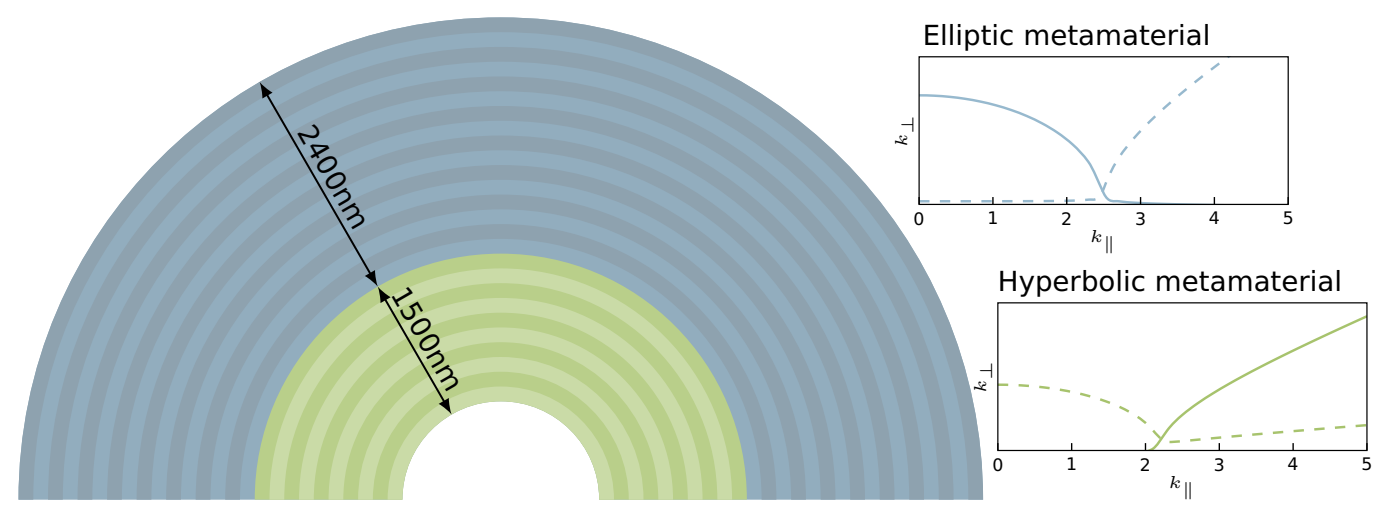

Figure 4. Design of two-part hyperlens. The insets show dispersion relations for both metamaterials (given by effective medium approximation): solid lines show the real part, while dashed lines show the imaginary part.

hyperlens magnification). However the beam width from a single point source is not small enough and this causes interference between beams formed by two sources for small separations. Below $300 \mathrm{~nm}$ we can not reliably measure the source separation. However, even at that limit it is obvious that with $715 \mathrm{~nm}$ operating wavelength the hyperlens is able to resolve details $\lambda / 2.4$, substantiating our claim of sub-wavelength imaging.

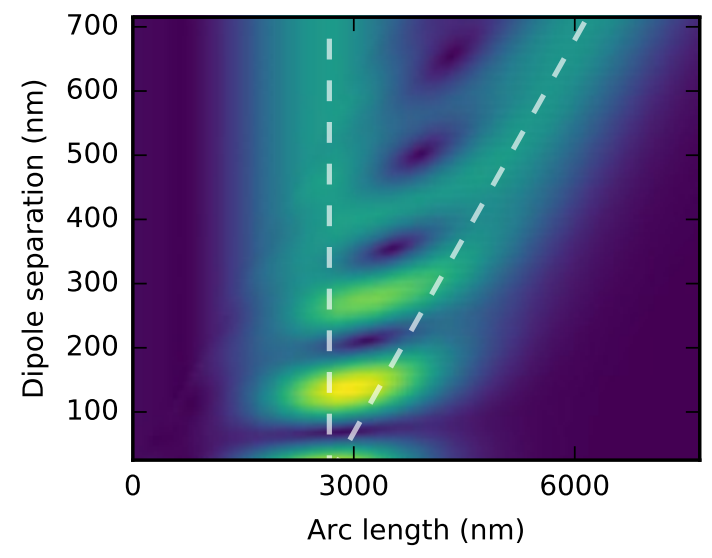

(a)

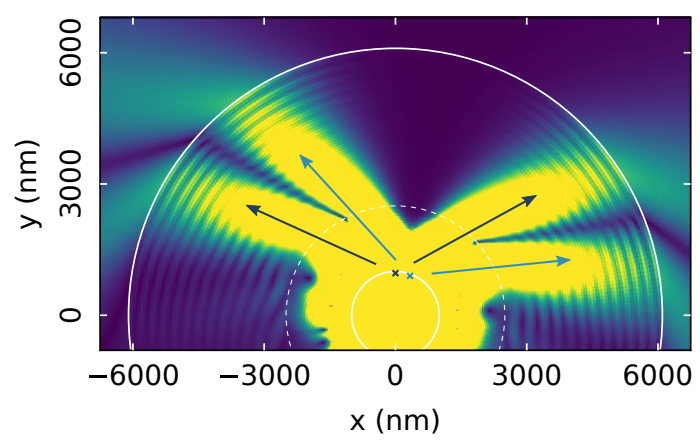

(b)

Figure 5. a) Fields on the outer interface of the hyperlens from point sources with varying distance. There are interference effects below $300 \mathrm{~nm}$ which makes the operation of the device unreliable. However, the device is fairly reliable above 300 $\mathrm{nm}$. The white dashed lines show where the peaks from the point sources should be without the interference effects.b) A simulation for case where the source separation is $350 \mathrm{~nm}$. Two sets of arrows indicate beams originating from the two point sources.

Finally, in figure 6 we demonstrate benefits of dark-field sub-wavelength imaging. When imaging dielectric scatterers using a bright-field hyperlens we see that the contrast between scattered and background fields is very low and distinguishing the objects in the output image is very difficult. On the other hand, when using a dark-field hyperlens for the same objects we see that the background radiation is filtered and scattered fields are clearly distinguishable in the output.

\section{CONCLUSIONS}

We have demonstrated the concept of a dark-field hyperlens: that is the device is able to resolve objects below diffraction limit while offering a good image contrast even for weakly scattering objects. We showed that the 


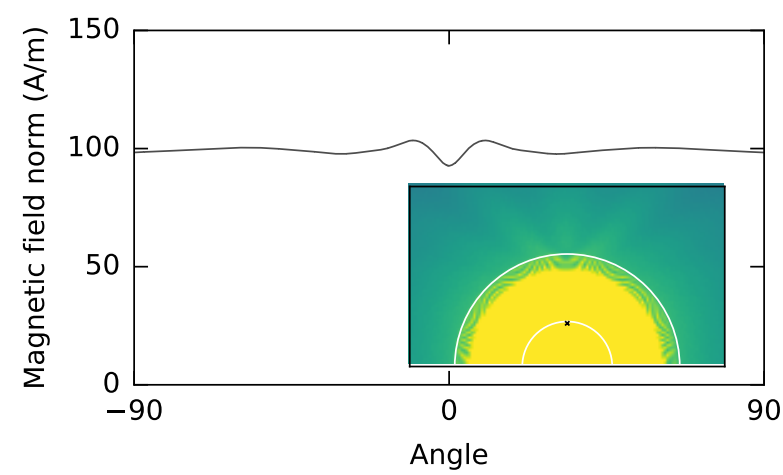

(a)

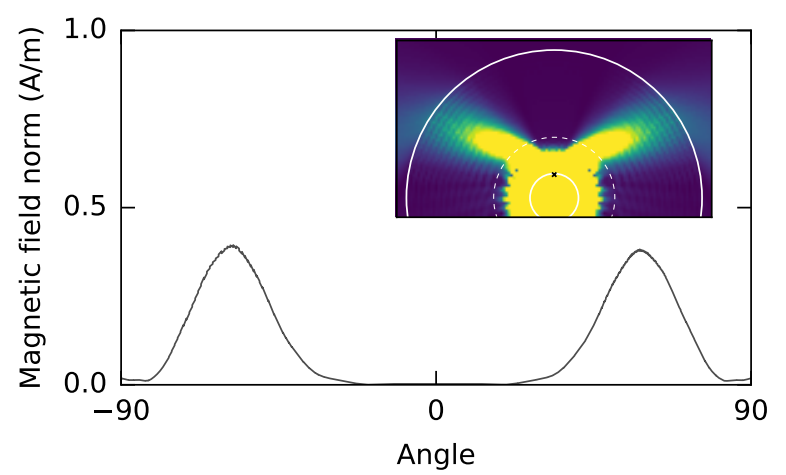

(b)

Figure 6. Comparison between bright- and dark field hyperlens for both metallic and dielectric scatterers. Plots show fields at the outer interface of the hyperlens. On the insets a cross marks the location of the dielectric scatterer. For bright-field hyperlens (a) it can be seen that the scattered fields are hardly visible against background fields. However, for dark-field hyperlens (b) there are no difficulties in distinguishing the scattered fields.

dark-field hyperlens is able to distinguish even weak scatterers $(n=1.5)$, whereas the image from usual brightfield hyperlens was dominated by strong background fields, so that weak scatterers were not distinguishable in the output.

As we demonstrated, our device allowed to distinguish objects separated by $\sim 300 \mathrm{~nm}$, which with $715 \mathrm{~nm}$ wavelength we used means $\sim \lambda / 2.4$ resolution. This is below usual $\sim \lambda / 2$ available to conventional optical microscopes and in theory could be improved further. The resolution is mainly limited by losses in the metamaterial. These losses might be lowered by either designing the hyperlens for a less lossy metal or by choosing different dielectric and metal volume filling fraction to reduce the imaginary component of the effective permittivity tensor components.

\section{ACKNOWLEDGMENTS}

This work has received support from Archimedes Foundation (Kristjan Jaak scholarship).

\section{REFERENCES}

[1] Cai, W. and Shalaev, V., [Optical Metamaterials: Fundamentals and Applications], Springer (2009).

[2] Poddubny, A., Iorsh, I., Belov, P., and Kivshar, Y., "Hyperbolic metamaterials," Nature Photonics 7(12), 948-957 (2013).

[3] Pendry, J. B., "Negative refraction makes a perfect lens," Phys. Rev. Lett. 85, 3966-3969 (Oct 2000).

[4] Jacob, Z., Alekseyev, L. V., and Narimanov, E., "Optical Hyperlens: Far-field imaging beyond the diffraction limit.," Optics express 14(18), 8247-8256 (2006).

[5] Liu, Z., Durant, S., Lee, H., Pikus, Y., Xiong, Y., Sun, C., and Zhang, X., "Experimental studies of far-field superlens for sub-diffractional optical imaging," Optics Express 15(11), 6947-6954 (2007).

[6] Smolyaninov, I. I., Hung, Y.-J., and Davis, C. C., "Magnifying superlens in the visible frequency range," Science 315(5819), 1699-1701 (2007).

[7] Kidwai, O., Zhukovsky, S. V., and Sipe, J. E., "Effective-medium approach to planar multilayer hyperbolic metamaterials: Strengths and limitations," Physical Review A - Atomic, Molecular, and Optical Physics 85(5), 1-12 (2012).

[8] Belov, P. A. and Hao, Y., "Subwavelength imaging at optical frequencies using a transmission device formed by a periodic layered metal-dielectric structure operating in the canalization regime," Physical Review B Condensed Matter and Materials Physics 73(11), 1-4 (2006). 
[9] Ikonen, P., Simovski, C., Tretyakov, S., Belov, P., and Hao, Y., "Magnification of subwavelength field distributions at microwave frequencies using a wire medium slab operating in the canalization regime," Applied Physics Letters 91(10), 2005-2008 (2007).

[10] Benisty, H. and Goudail, F., "Dark-field hyperlens exploiting a planar fan of tips," Journal of the Optical Society of America B 29(9), 2595 (2012).

[11] Guo, Y., Newman, W., Cortes, C. L., and Jacob, Z., "Applications of hyperbolic metamaterial substrates," Advances in OptoElectronics 2012(1), 1-10 (2012).

[12] Repän, T., Lavrinenko, A. V., and Zhukovsky, S. V., "Dark-field hyperlens: Super-resolution imaging of weakly scattering objects," Optics Express 23, 25350 (sep 2015). 